\title{
МОДЕЛИРОВАНИЕ И АНАЛИЗ ТОЧНОСТИ АЛГОРИТМОВ ШУМОПЕЛЕНГАЦИИ ИСТОЧНИКОВ НИЗКОЧАСТОТНЫХ ГИДРОАКУСТИЧЕСКИХ СИГНАЛОВ В МЕЛКОМ МОРЕ
}

\author{
Ю. В. Махнев \\ АО «Научно-исследовательский институт «Атолл»
}

Поступила в редакцию 30.08.2019 г.

\begin{abstract}
Аннотация. При пространственной обработке низкочастотных гидроакустических сигналов в мелком море возникают ошибки пеленгации, связанные с многомодовым характером распространения гидроакустических сигналов. Для уменьшения ошибок пеленгации необходимо использовать алгоритмы шумопеленгации, согласованные со средой распространения сигналов. Из-за сложности их реализации на практике используются адаптивные, квазиоптимальные алгоритмы. Однако, при большой дисперсии фазовых скоростей нормальных волн и приёмной антенне с малой разрешающей способностью, эффективность алгоритмов уменьшается. В работе проведено моделирование нескольких известных алгоритмов шумопеленгации, в том числе полностью согласованного со средой. В работе исследована зависимость точности пеленгования при применении разных алгоритмов от способности гидроакустической системы разрешать нормальные волны. Показано, что их разрешение зависит от периода интерференции, длины антенны и начальных фаз нормальных волн. Приведены формулы и соотношения, позволяющие оценить величину ошибки определения пеленга. Проведено моделирование работы гидроакустической системы с конкретными параметрами. Параметры волновода задавались близкими к реальным. Рассчитаны математическое ожидание и среднее квадратичное отклонение ошибок пеленгации и вероятность ложного обнаружения источника звука.

Ключевые слова: гидроакустика, согласованная со средой обработка в низкочастотном диапазоне, квазиоптимальные алгоритмы, мелкое море, линейные антенны, пеленгование, шумопеленгация, разрешение сигналов.
\end{abstract}

\section{ВВЕДЕНИЕ}

Для обнаружения и пеленгования низкочастотных источников звука в пассивном режиме используются дискретные, линейные гидроакустические антенны, состоящие из ненаправленных приёмников одинаковой чувствительности. Параметры этих антенн, такие как протяженность, межэлементное расстояние и количество приёмников, выбирают в соответствии с рабочим диапазоном частот, требуемым коэффициентом усиления антенны, периодом пространственной когерентности сигнала и другими критериями. Направленные свойства таких антенн и возможность определения точного направления на источник звука в условиях мелкого моря

(C) Махнев Ю. В., 2019 изучались в множестве работ [1-4 и их списки литературы]. В мелком море количество нормальных волн не велико (от 1 до 10), однако разброс значений их фазовых скоростей может достигать нескольких сотен м/с, что приводит к большим ошибкам пеленгования.

Во всех приведенных работах отмечается, что оценка пеленга на источник звука определяется с ошибкой из-за явления дисперсии звука и многомодового распространения в волноводе. В частности, в [1] показано, что при определенном соотношении между длиной волны интерференции и межэлементным расстоянием антенны нормальные волны в отклике антенны проявляются в разных направлениях как отдельные максимумы. Направление прихода нормальных волн $\theta$ относительно волны, на которую настроен фазовый компенсатор, определяется исходя из соотношения их фазовых скоростей. 
Моделирование и анализ точности алгоритмов шумопеленгации источников низкочастотньх ...

$$
\theta=\arccos \left(\frac{c_{k} \cdot \cos \left(\theta_{u c m}\right)}{c_{k}}\right),
$$

где $c_{k}, c_{m}$ фазовые скорости компенсатора и $m$-й нормальной волны соответственно, $\theta_{u c m}$ истинное направление на источник звука.

Из (1) следует вывод, что для получения пеленга в истинном направлении на источник звука необходимо согласовать фазовую скорость в компенсаторе с фазовой скоростью нормальной волны. В [3] указываются ссылки на исследования возможных искажений и эффектов в отклике антенны при наличии рассогласования компенсатора со средой распространения. К искажениям относят расширение, расщепление и смещение оценки пеленга основного максимума, возрастание бокового поля. Если согласование со средой не обеспечено, при пространственной обработке возникает ошибка пеленгации, пропорциональная дисперсии фазовых скоростей нормальных волн, и в мелком море может достигать $35^{\circ}$.

Особое внимание следует обратить на тот факт, что линейные антенны очень чувствительны к рассогласованию со средой распространения при обработке сигналов, приходящих с направлений, близких к направлению бегущей волны и при ошибке пеленгации величиной в несколько десятков градусов, по сути имеют слепую зону.

Анализ результатов предшествующих работ

В [4] авторы провели объемные исследования по работе линейной антенны в условиях мелкого моря при отсутствии согласования со средой. В данной работе принимаемые сигналы на антенну представлялись в виде суммы плоских волн, параметры которых в точке приёма определялись согласно передаточной функции, рассчитанной по алгоритму KRAKEN. Волновод был представлен тремя нормальными волнами. Приём осуществлялся на 16-и элементную антенну. При моделировании параметры сигналов менялись в широких пределах, рассматривался диапазон частот от 6 до 120 Гц, направления приёма сигналов от нормали к антенне до направления бегущей волны, глубина излучателя до 100 м, расстояние источник-антенна от 500 м до 9 км. Кроме этого, приведены результаты по точности пеленгования в зависимости от расстояния между приёмниками (от 1 до 30 м).

Эти исследования показывают, что в подавляющем большинстве вариантов, приведенных выше параметров приёма сигналов, ошибка пеленгования в направлениях \pm 40 градусов от нормали к антенне составляет $\leq 5$ градусов. Для направлений бегущей волны антенны ошибка пеленгования достигает 25 градусов.

На сегодняшний день существует великое множество работ, посвященных исследованию и синтезу различных алгоритмов пеленгования сигналов для разных условий и антенн. Наиболее известные алгоритмы хорошо обобщены и представлены в [5]. При многомодовом приёме сигналов, характерном для мелкого моря, для повышения точности пеленгования необходимо учитывать в алгоритмах априорную информацию о среде распространения. Такие алгоритмы получили в литературе название согласованные со средой распространения (англ. «matched-field processing»).

Наряду с предполагаемыми выигрышами от согласованной обработки в виде более точного пеленгования или повышения отношения сигнал/шум появляется масса трудностей при реализации такой обработки, связанные с нестационарностью передаточной функции среды, сложной сигнально-помеховой обстановкой и т. п. Состояние развития согласованной обработки показывают обзорные статьи $[6,7]$. Авторы обзора [6] отмечают, что множество исследователей ставят под сомнение практическую целесообразность согласованной обработки и даже саму возможность корректного решения задачи, но в тоже время это подталкивает исследователей к разработке робастных алгоритмов, которые при разумных требованиях к потери точности допускали бы определенное рассогласование.

При этом есть ряд научных исследований и результатов обработки экспериментальных данных [12], в которых, несмотря на использование квазиоптимальных алгоритмов, учитывающих априорное знание о дисперсионных свойствах фазовых скоростей нормаль- 


\section{Ю. В. Махнев}

ных волн и несмотря на работу в узкой полосе частот при отношении сигнал/шум свыше 20 дБ, оценка пеленга является смещенной и имеет дисперсию $\sim 5-7^{\circ}$. Возникает естественный вопрос: с чем связана ошибка оценки пеленга?

\section{Постановка задачи}

В связи с недостаточной точностью пеленгования источников квазиоптимальными алгоритмами, при условии, что шумы различной природы можно считать незначительными и параметры среды распространения сигналов известны точно, необходимо решить ряд научно-исследовательских задач:

- Определить причину возникновения ошибок пеленгации.

- Произвести оценку математического ожидания и дисперсии ошибок пеленгации известными алгоритмами, для условий мелкого моря и работы в низкой полосе частот.

- Определить зависимость ошибок пеленгации от направления прихода сигнала на антенну.

\section{МАТЕРИАЛЫ И МЕТОДЫ ИССЛЕДОВАНИЯ}

Для выполнения поставленных задач используются методы системного анализа и управления, статистической обработки информации, теории вероятности, теории цифровой обработки сигналов. Анализ подкреплён результатами моделирования.

\section{Модель волновода и сигналов}

Опишем принятую в данной работе модель формирования гидроакустических сигналов на приёмниках антенны. Волновод считается горизонтально-однородным с постоянной скоростью распространения звука равной 1500 м/с. Изменение параметров волновода в зависимости от глубины не рассматриваются. При моделировании считаем, что амплитуды нормальных волн в точке приёма соответствуют некоторым глубинам источника и приёмника, которые априорно известны. Приёмная антенна состоит из $N$ приёмников с межэлементным расстоянием $d$, которые имеют координаты $\left(x_{n}, y_{n}\right)$, где $n$ - номер приёмника. Центр антенны расположен в точке $\left(x_{0}, y_{0}\right)$. Горизонтальное расстояние между источником звука и фазовым центром антенны обозначим как $R$. Считаем, что источник находится в дальнем поле антенны и его скорость равна нулю. Пеленг на источник определяется относительно оси антенны и обозначается $\theta_{\text {ис }}$, при этом направление $90^{\circ}$ соответствует нормали к антенне, а $0^{\circ}$ направлению бегущей волны.

Будем считать, что источник звука излучает непрерывный гармонический сигнал на заданной частоте $f$. Для моделирования сигнала в точке приёма используем плосковолновую модель, тогда отсчеты сигнала, формируемые на приёмнике с номером n вычисляются по формуле (2).

$$
P(n)=\sum_{m} A_{m} \cdot e^{-j \frac{2 \pi f}{c_{m}} \cdot r(n)},
$$

где $A_{m}$ и $c_{m}$ - амплитуда и фазовая скорость нормальной волны, $n=1 . . N$ номера приёмников, $r(n)=R+x_{n} \cdot \cos \left(\theta_{u c m}\right)+y_{n} \cdot \sin \left(\theta_{u c m}\right), f-$ частота излучения, $j$ - мнимая единица.

Количество источников сигнала при анализе всегда задаём равным единице. Так как нас интересуют ошибки пеленгации, связанные только с взаимодействием нормальных волн, полагаем шумы моря и различные искажения сигналов из-за технических факторов незначительными. Любое значение акустического поля на приёмниках антенны отличное от нуля создаётся источником звука. При использовании алгоритмов, согласованных со средой, считаем, что параметры нормальных волн известны точно. Таким образом, при дальнейшем анализе алгоритмов, ошибки пеленгации будут зависеть только от параметров антенны и особенностей алгоритма обработки.

Моделирование проводилось в MATLAB R2014b, 64-bit. Все вычисления и представление данных производились в типе double.

Алгоритмь обработки гидроакустических сигналов

Все рассматриваемые алгоритмы используют функционал вычисления отношения правдоподобия, результатом которого является отклик антенны, максимум которого указывает направление на источник звука. Рассмотрим их. 
Моделирование и анализ точности алгоритмов шумопеленгации источников низкочастотных ...

(А) Приёмник на основе модели с эквивалентной плоской волной.

Одним из самых простых для реализации и самых известных алгоритмов, является алгоритм на основе модели с эквивалентной плоской волной. Его реализация и характеристики представлены в [4 и 8]. Отклик антенны, получаемый этим алгоритмом вычисляется по формуле

$$
D(\theta)=\sum_{n} P(n) \cdot H(n, \theta),
$$

где

$$
H(n, \theta)=e^{j \frac{2 \pi f}{c_{k}} \cdot\left[x_{n} \cdot \cos (\theta)+y_{n} \cdot \sin (\theta)\right]}
$$

$\theta$ - направление наблюдения, $H(n, \theta)$ - компенсатор, обеспечивающий оптимальную пространственную фильтрацию сигнала, поступающего из направления $\theta$ для приёмника с номером $n, c_{k}$ - фазовая скорость, на которую настроен компенсатор.

Данный алгоритм не использует никакой априорной информации о среде распространения сигнала, кроме $c_{k}$ - которая выбирается равной скорости распространения звука.

(Б) Приёмник на основе модели с эквивалентной плоской волной с использованием эффективной фазовой скорости.

Анализ и реализация данного алгоритма представлена в [3]. Алгоритм реализуется аналогично алгоритму (А) с той лишь разницей, что фазовая скорость компенсатора вычисляется согласно выражению

$$
c_{k}=\frac{\sum_{m} c_{m} \cdot A_{m}^{2}}{\sum_{m} A_{m}^{2}} .
$$

Идея алгоритма состоит в том, чтобы настроить компенсатор на некую «среднюю» фазовую скорость присутствующих на данной частоте нормальных волн и за счет этого получить более точные оценки пеленга, чем в алгоритме (A).

(B) Некогерентное суммирование откликов антенны на отдельные нормальные волны

Идея использования этого алгоритма высказывалась в [2]. В основе данного алгоритма лежит объединение откликов антенны на различные нормальные волны без учета их фаз, т. е. путём их энергетического суммиро- вания. В этом случае отклик антенны рассчитывается по формуле

$$
D(\theta)=\sum_{m}\left|\sum_{n} P(n) \cdot H(n, \theta, m)\right|^{2},
$$

В отличие от компенсатора $H$ в алгоритме (А) и (Б) здесь в качестве фазовой скорости используется фазовая скорость $m$-й нормальной волны и учитывается её амплитуда.

$$
H(n, \theta, m)=A_{m} \cdot e^{j \cdot \frac{2 \pi f}{c_{m}} \cdot\left[x_{n} \cdot \cos (\theta)+y_{n} \cdot \sin (\theta)\right]} .
$$

(Г) Когерентное объединение откликов антенны на отдельные нормальные волны

Данный алгоритм также представлен в [8] и является оптимальным, при условии, что все параметры источника, приёмников и передаточной функции волновода известны точно. Именно к реализации этого алгоритма стремится общество исследователей-гидроакустиков. Суть алгоритма заключается в создании такого компенсатора $H$, который полностью совпадает с принимаемым звуковым полем (2).

$$
D(\theta)=\sum_{n} P(n) \cdot \sum_{m}\left(H(n, \theta, m) \cdot e^{j \cdot \frac{2 \pi f}{c_{m}} \cdot R}\right),
$$

Алгоритм (6) отличается от алгоритма (5) объединением откликов антенны на отдельные нормальные волны с учетом их начальных фаз. На практике пеленг определяют по энергии отклика антенны, что подразумевает вычисление $|D(\theta)|^{2}$. И в этом случае, при определенных соотношениях даже алгоритм $\Gamma$ даёт ошибку пеленгации, что будет рассмотрено ниже.

Отклики антенны, получаемые согласно представленным алгоритмам, могут содержать несколько максимумов, сопоставимых по амплитуде, которые появляются из-за наличия нескольких нормальных волн. В связи с этим появляется неоднозначность определения пеленга и встает вопрос о выделении значимых максимумов.

В рамках данной работы считаем, что значимыми максимумами $M$ являются те, которые по уровню больше 0,5 (-3дБ) от главного максимума отклика антенны $\max \left(|D|^{2}\right)$. Максимумы считаются разрешенными если величина провала между ними составляет не мень- 


\section{Ю. В. Махнев}

ше чем 0,5 (-3дБ) от меньшего из максимумов $M$. Данное условие упоминается в [5] и считается надежным при обнаружении первичных отметок автоматическим обнаружителем.

\section{РЕЗУЛЬТАТЫ ИССЛЕДОВАНИЙ И ИХ ОБСУЖДЕНИЕ}

Исследование влияния интерферениии нормальных волн на точность пеленгования

Рассматривая выражения для расчета откликов антенны алгоритмами (А-Г) можно заметить, что все они построены на единой операции перемножения и сложения значений $P$ и $H$. Следовательно, все свойства данной операции присущи каждому алгоритму. Чтобы понять, как влияет интерференция нормальных волн на получаемые отклики антенны в первую очередь подробно рассмотрим работу алгоритма (А).

В рамках рассматриваемой модели скорость звука в среде принимает значение $1500 \mathrm{~m} / \mathrm{c}$, тогда положим для алгоритма (3) $c_{k}=1500$. В ряде исследовательских работ Елисеевнина В. А. [1, 2 и др.] и в [9] было показано, что отклик антенны на сигнал, содержащий одну нормальную волну определяется по выражению

$$
D_{m}(\theta)=A_{m} \cdot e^{j \frac{2 \pi f}{c_{m}} \cdot R} \cdot \frac{\sin (x)}{x},
$$

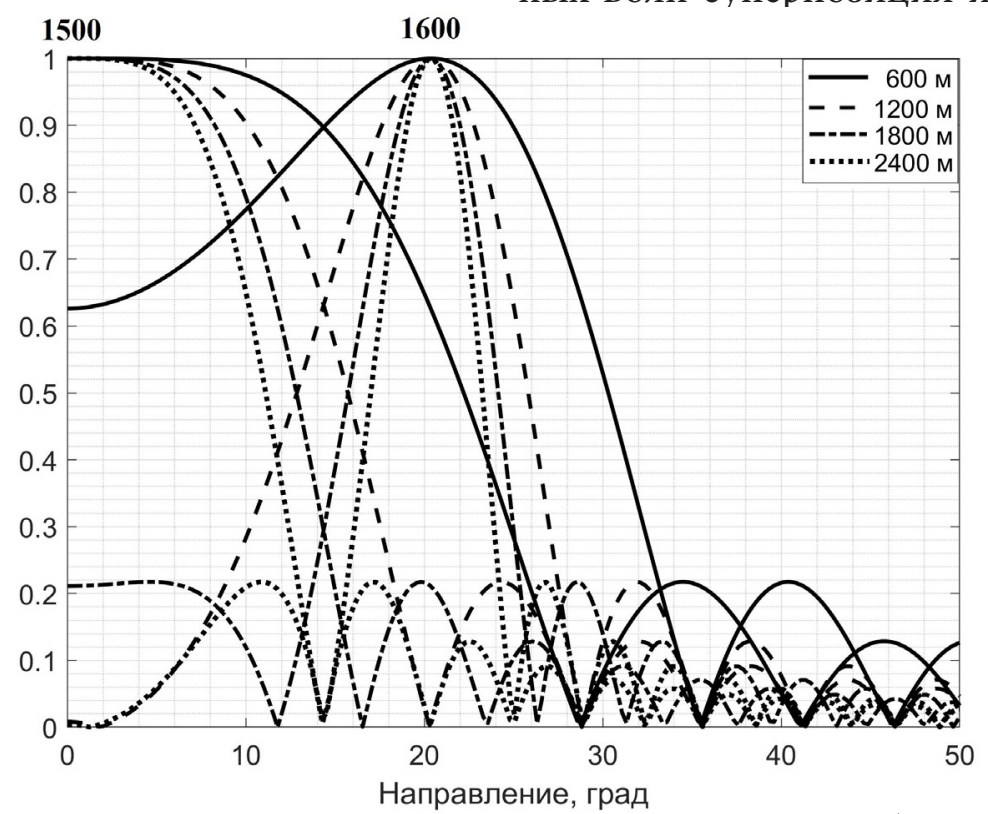

Рис. 1. Модуль откликов линейной антенны с различной апертурой $(600,1200,1800$ и 2400 м) и при значениях фбазовых скоростей нормальной волны 1500 и 1600 м/c где $x=\frac{\pi \cdot L_{0} \cdot f}{c_{k}} \cdot\left[\cos (\theta)-\cos \left(\theta_{m}\right)\right]$,

$L_{0}=(N-1) \cdot d-$ длина апертуры линейной антенны, $\theta_{m}$ - направление на $m$-ю нормальную волну, рассчитанное согласно (1).

Рассмотрим более детально получаемые отклики антенны согласно алгоритму А на конкретном примере. На рис. 1 представлены нормированные на максимум интенсивности модули откликов антенны (7) при приёме одной нормальной волны с фазовой скоростью 1500 и $1600 \mathrm{M} / \mathrm{c}$ на частоте 20 Гц с направления бегущей волны антенны при различных размерах апертуры её апертуры.

На рис. 1 явно видны свойства откликов антенны, которые указывались в [1 и 9]. Важно отметить, что основные лепестки функции (7) на разные нормальные волны могут пересекаться и при сложении $D_{1}(\theta)+D_{2}(\theta)$ немаловажную роль будет играть начальная фаза нормальных волн. Пусть нормальные волны имеют одинаковую амплитуду $A_{1}=A_{2}$, тогда на рис. 2 приведем модуль откликов линейной антенны на сигнал, являющийся суперпозицией двух нормальных волн при длине апертуры 1200 м. Отклики нормированы на максимум отклика на одну нормальную волну.

На данном примере видно, что в зависимости от разности начальных фаз нормальных волн суперпозиция их откликов сильно 


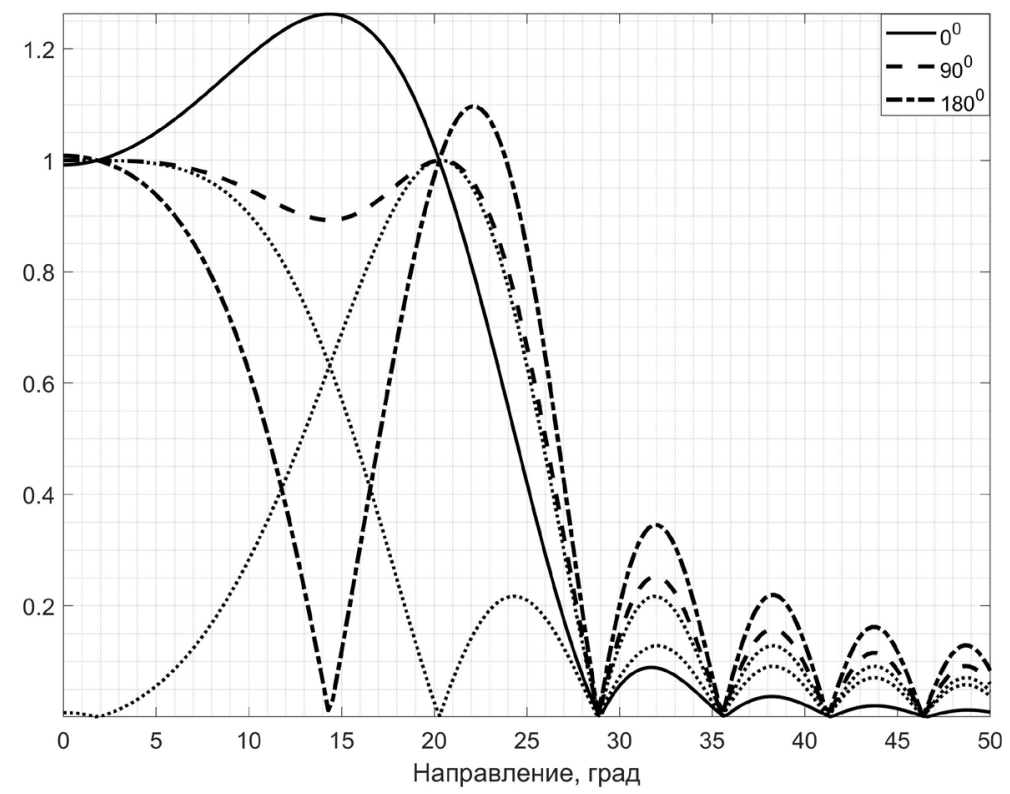

Рис. 2. Модуль откликов линейной антенны на сигнал содержащий две нормальных волньы с фазовыми скоростями 1500 и 1600 м/с и разностью начальных фаз $0^{\circ}$ (сплошная),

$90^{\circ}$ (пунктирная) и $180^{\circ}$ (штрихпунктирная), нормированные на максимум отклика одной нормальной волны. Длина апертуры 1200 м. Точками показаны отклики на нормальные волны по отдельности

отличается. Всё дело в том, что основные лепестки откликов на отдельные нормальные волны (рис. 2 точками) пересекаются, и чем больше это пересечение, тем большее влияние они оказывают друг на друга. Так при разности фаз 00 (рис. 2 сплошная) часть этих волн сложилась когерентно, что дало единственный максимум отклика в промежуточном направлении равном $\sim 15^{\circ}$. Это направление не совпадает ни с истинным направлением $0^{\circ}$ ни с направлением $\sim 20^{\circ}$ на нормальную волну с фазовой скоростью 1600 м/с. По мере увеличения разности начальных фаз в отклике антенны начинают постепенно выделяться максимумы, соответствующие нормальным волнам. При разности фаз $90^{\circ}$ стали заметны два максимума (рис. 2 пунктирная). Далее глубина провала между максимумами увеличивается и при разности фаз $180^{\circ}$ достигает нуля (рис. 2 штрихпунктирная). Несмотря на то, что в этом случае максимумы, указывающие на наличие двух нормальных волн, определяются однозначно, их направление смещено относительно истинного. Направление на нормальную волну с фазовой скоростью 1600 м/с в этом случае составляет $\sim 22^{\circ}$.
Из приведенного выше анализа можно сделать вывод, что в зависимости от степени перекрытия основных лепестков откликов на нормальные волны и их начальных фаз зависит точность пеленгования.

Рассмотрим от чего зависит изменение начальных фаз нормальных волн. Выражение (2) есть ничто иное как сложение нескольких одинаково направленных гармонических колебаний с близкими пространственными частотами. Результат такой суммы называют биениями и говорят, что волны интерферируют. Биения хорошо изучены и рассматриваются в массе литературных источников, например, в [10]. Однако, литературы в которой рассматривается влияние биений пространственных волн на эффективность работы антенн не было найдено. В связи с этим, приведём основные соотношения, возникающие в результате биений, модифицируя материал, изложенный в [10].

При сложении двух нормальных волн, согласно выражению (2), амплитуда результирующего колебания будет равна

$$
A(r)^{2}=A_{1}^{2}+A_{2}^{2}+2 A_{1} A_{2} \cos (\Delta k \cdot r)
$$

а направление $\bar{\theta}$ максимума отклика антенны на этот сигнал будет средним направлени- 


\section{Ю. В. Махнев}

ем между направлениями на нормальные волны определенные по (1).

$$
\begin{gathered}
\bar{\theta}=\arccos \left(\frac{c_{k} \cdot \bar{k} \cdot\left(1+\frac{1}{2} \cdot \frac{A_{2}-A_{1}}{A_{1}+A_{2}} \cdot \frac{\Delta k}{\bar{k}}\right)}{2 \pi f}\right), \\
\Delta k=2 \pi f \cdot \cos \left(\theta_{u c m}\right) \cdot\left(\frac{1}{c_{1}}-\frac{1}{c_{2}}\right), \\
\bar{k}=\pi f \cdot \cos \left(\theta_{u c m}\right) \cdot\left(\frac{1}{c_{1}}+\frac{1}{c_{2}}\right)
\end{gathered}
$$

где $A_{1}, A_{2}, c_{1}, c_{2}$ - амплитуды и фазовые скорости нормальных волн, $c_{k}-$ фазовая скорость компенсатора, $f$ - несущая частота, $r$ - текущее расстояние (аналог времени).

Период интерференции $T R$ определяется по известной формуле $T R=\frac{1}{f \cdot\left(\frac{1}{c_{1}}-\frac{1}{c_{2}}\right)}$, а период результирующего колебания (2) при двух нормальных волнах $T K=2 \cdot T R$.

Представим результирующее колебание на рис. 3.

Из рис. 3 и выражения (8) видно, что амплитуда результирующего колебания зависит от разности волновых чисел нормальных волн и пройденного расстояния $r$. В точках где функция (8) пересекает ось $r$ происходит переворот фазы на $\pi$. Таким образом, результирующее колебание имеет полную аналогию с сигналами с амплитудной модуляцией с подавлением несущей частоты [11].

Идея использования этих сигналов состоит в том, что несущая частота АМ-сигнала подавляется за счет того, что несущая частота в разных половинах периода $T K$ находится в противофазе. В случае рассмотрения сигнала (2), состоящего из двух нормальных волн одинаковой амплитуды и с фазовыми скоростями 1500 и 1600 м/с на частоте 20 Гц период $T K=2400$ м. Из-за ограниченной протяженности проекции линейной антенны на направление наблюдения, интервал анализа этого сигнала ограничивается величиной $L=L_{0} \cdot \cos \left(\theta_{u c m}\right)$. В связи с этим в большинстве случаев длина проекции антенны $L$ оказывается значительно меньшей, чем период сигнала $T K$. В работе [12] часть сигнала, фиксируемого антенной, названа волновым пакеmом, тем самым подчеркивая пространственный характер изменчивости этого сигнала, и то что сигнал является лишь частью более сложного модулированного сигнала.

Вышесказанное можно связать с поведением отклика антенны на рис. 2. $(L=0,5 \cdot T K)$. Имеет большое значение какая часть периода

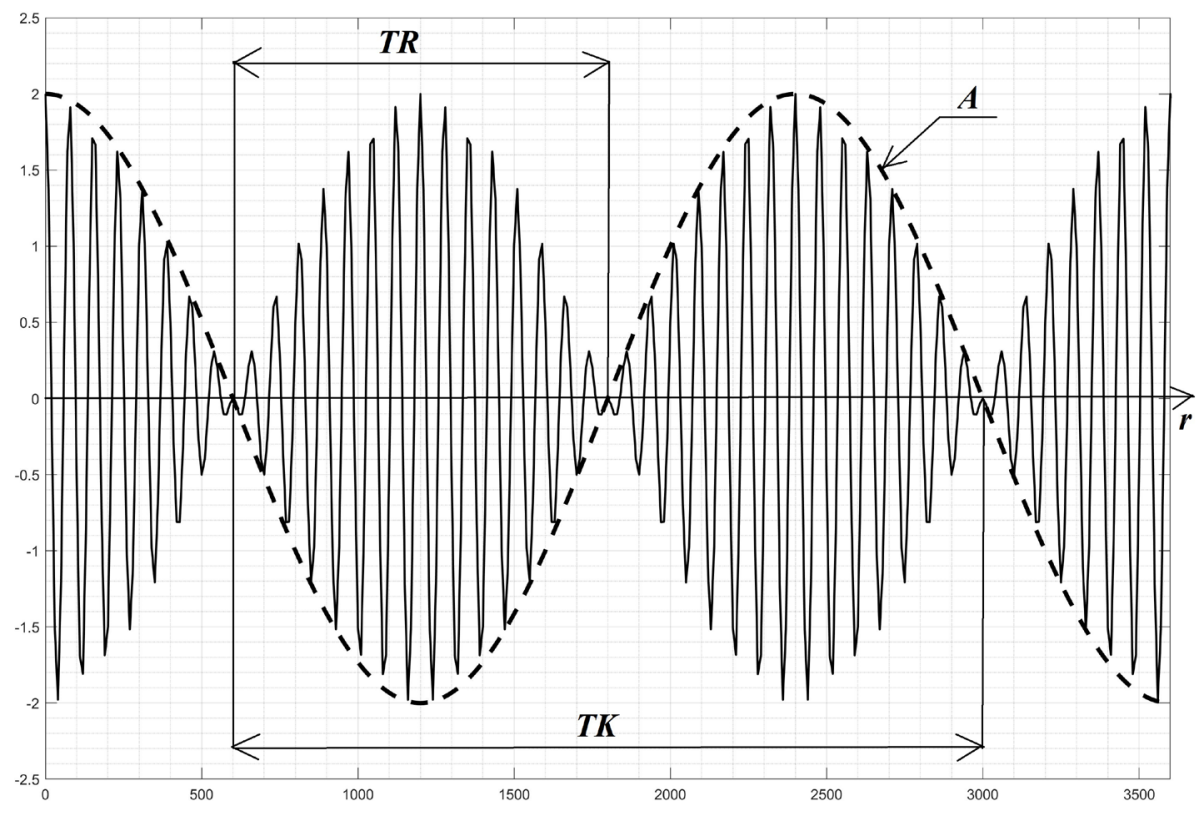

Рис. 3. Результат сложения двух нормальных волн одинаковой амплитуды с разными фазовыми скоростями 
Моделирование и анализ точности алгоритмов шумопеленгации источников низкочастотных ...

$T K$ содержится в волновом пакете. Для случая нулевой разности фаз будет фиксироваться волновой пакет TR (рис. 3), т. е. только первая или только вторая половина $T K$. В этом случае подавления несущей АМ-сигнала не происходит, что проявляется в виде отклика антенны в промежуточном направлении согласно (9) (рис. 2 сплошная). Разность фаз $90^{\circ}$ соответствует приёму $0,75 \cdot T R$ из одной половины $T K$ и $0,25 \cdot T R$ из второй половины. Таким образом произошло частичное подавление отклика в промежуточном направлении (рис. 2 пунктирная). Разность фаз $180^{\circ}$ соответствует захвату половины $T R$ из левой и правой части $T K$, что обеспечило полное подавление и наибольшую глубину провала между максимумами.

Обобщим вышесказанное для более общего случая. Если величина $L \geq T K$ отклик антенны будет содержать несколько разделенных максимумов, соответствующих количеству нормальных волн. Разность фаз нормальных волн будет незначительно влиять на их положение.

Для случаев $L<T K$ результат будет зависеть от разности фаз нормальных волн или от того, какую часть периода $T K$ содержит волновой пакет. Рассмотрим частный случай, при котором имеются две нормальные волны одинаковой амплитуды. В этом случае волны интерферируют наиболее сильно и при определенных условиях могут даже сложиться в противофазе. Поэтому случай $L<T K$ можно разделить на три возможных ситуации:

1. Волновой пакет содержит информацию только из одной половины $T K$ или захватывает малую часть другой половины, недостаточную для компенсации отклика в промежуточном направлении $\bar{\theta}$. Отклик антенны будет иметь один максимум, направление которого соответствует (9). Методическая ошибка пеленга будет равна $\Delta \theta=\theta_{u c m}-\bar{\theta}$.

2. Волновой пакет содержит информацию из разных половин $T K$, причем соотношение захваченных частей достаточно близко к отношению $1: 1$, для подавления $\bar{\theta}$ и образования провала между двумя максимумами. В этом случае появляется неопределенность при оценке пеленга, так как существует два максимума. Ошибка пеленгации носит сложный характер и зависит от разности фазовых скоростей нормальных волн, длины антенны, частоты и направления прихода сигнала.

3. Волновой пакет содержит информацию из разных половин $T K$, соотношение частей близко к 1:1 и при этом $L<<T K$. Случай характеризуется пересечением основных лепестков квадратов модулей откликов антенны на две нормальные волны по уровню $>0,5$. В этом случае имеет место интерференционный минимум, который в данной работе отвечает условию $\frac{\max \left(|D|^{2}\right)}{\max \left(\left|D_{m}\right|^{2}\right)}<0,5$, т. е. более половины энергии нормальных волн поглощено в интерференции.

Стоит отметить, что в зависимости от параметров ГАС, разности фазовых скоростей и от начальных фаз нормальных волн на периоде $T K$ могут иметь место все три вышеописанных ситуации. Наличие той или иной ситуации на периоде можно выразить в процентном отношении в зависимости от уровня пересечения $\gamma$ основных лепестков модулей откликов антенны на нормальные волны. Определить этот уровень можно по выражению

$$
\gamma=\left\{\begin{array}{l}
\left|\frac{\sin (x)}{x}\right|^{2}, 0<x<\pi \\
0, x \geq \pi
\end{array},\right.
$$

где

$$
x=\frac{\pi \cdot L_{0} \cdot f}{c_{1}}\left[\cos (\bar{\theta})-\cos \left(\theta_{1}\right)\right] .
$$

Уровень равный нулю, означает отсутствие пересечения. Ниже приведем таблицу, в которой представим процентное содержание на периоде $T K$ той или иной ситуации. Ситуацию с разрешением, т. е. с наличием провала между откликами на нормальные волны, обозначим $T R_{\theta}$, ситуацию в которой разрешение откликов отсутствует $T R_{\bar{\theta}}$, а наличие интерференционного минимума $T R_{\min }$.

Как видно из табл. 1, уровень перекрытия квадратов модулей откликов на нормальные волны $\gamma$ определяет в каком процентном соотношении отклик антенны будет иметь один значимый максимум в направлении $\bar{\theta}$ или два максимума в случае $T R_{\theta}$. Каждая ситуа- 
Таблица 1

Зависимость процентного соотношения наличия/отсутствия разрешения на периоде ТК основных лепестков квадратов модулей откликов на две нормальные волны одинаковой амплитуды от уровня их перекрытия $\gamma$

\begin{tabular}{|c|c|c|c|c|c|c|c|c|c|}
\hline$\gamma$ & 0 & 0,1 & 0,2 & 0,3 & 0,4 & 0,5 & 0,6 & 0,7 & 0,8 \\
\hline$T R_{\theta}, \%$ & 100 & 98 & 77 & 66 & 58 & 48 & 41 & 30 & 4 \\
\hline$T R_{\bar{\theta}}, \%$ & 0 & 2 & 23 & 34 & 42 & 51 & 58 & 66 & 72 \\
\hline$T R_{\min }, \%$ & 0 & 0 & 0 & 0 & 0 & 1 & 1 & 4 & 24 \\
\hline
\end{tabular}

ция содержит свою методическую ошибку пеленгации.

Отсюда можно сделать важный вывод: если ситуация $T R_{\bar{\theta}}$ в большем процентном соотношении, то методическую ошибку следует устранить путём настройки компенсатора на фазовую скорость волны, равную $c_{k}^{\prime}=\frac{c_{k} \cdot \cos \left(\theta_{u c m}\right)}{\cos (\bar{\theta})}$. Данная ситуация характерна для $\gamma>0,5$ и возникает при наличии источника звука в направлениях близких к нормали или для коротких антенн. В противном случае, компенсатор следует настраивать на истинную фазовую скорость нормальной волны.

Сделанный вывод поясняет алгоритм Б. Алгоритм Б будет более эффективен если ситуация $T R_{\bar{\theta}}$ встречается чаще, иначе более эффективной будет настройка компенсатора на фазовую скорость наиболее интенсивной нормальной волны.

Из приведённого анализа можно сделать вывод о том, что алгоритмы А-В будут давать ошибку пеленга, даже с учетом большого с/ш и точных априорных знаний о среде, ввиду того что не учитывают взаимное влияние нормальных волн, вызванных недостаточным разрешением антенной системы.

Реальный гидроакустический волновод в большинстве случаев содержит более двух нормальных волн, причем в зависимости от глубины и расстояния до источника меняются их амплитуды. Для таких ситуаций провести аналитический анализ довольно сложно, поэтому далее ограничимся рассмотрением моделирования работы конкретных вариантов ГАС.
Получение оценки точности пеленгования для антенны, работающей в условиях, приближенных к реальности.

Произведем оценку точности пеленгования различными алгоритмами (А-Г) для нормальных волн, дисперсионные кривые фазовых скоростей которых зададим в соответствии с оценками, полученными по данным натурного эксперимента в одном из районов Баренцева моря [13]. Представим их на рис. 4.

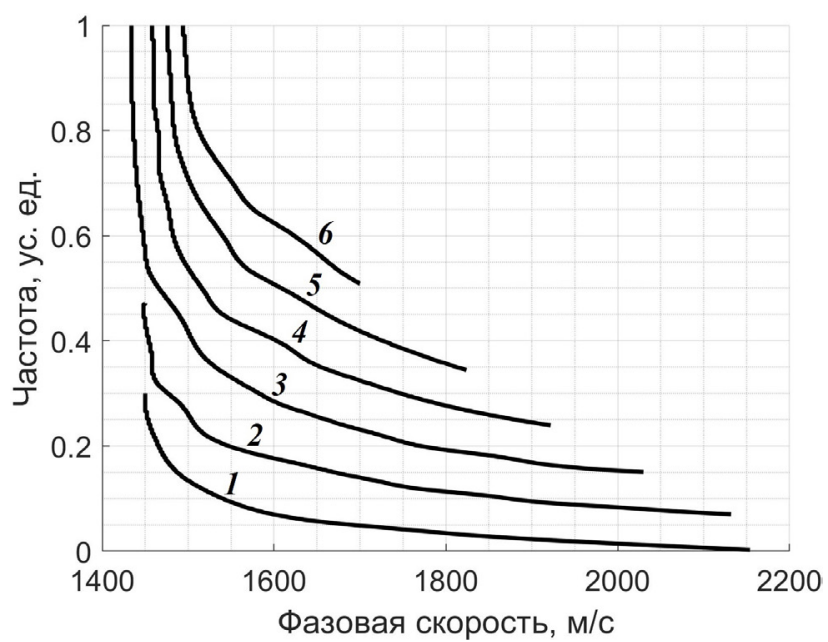

Рис. 4. Дисперсионные кривые фазовых скоростей нормальных волн, полученные с использованием импульсных методов калибровки волновода в Барениевом море при глубине излучателя 30 м. Цифрами пронумерованы нормальные волны

Отметим, что нормальные волны с номерами 2, 3 и 4 несут в себе основную энергию и их относительные амплитуды находятся в диапазоне от 0,8 до 1, что близко к рассмотренному варианту нормальных волн с одинаковыми амплитудами в разделе 3. По отношению к энергонесущим нормальным волнам, 
Моделирование и анализ точности алгоритмов шумопеленгации источников низкочастотньх ...

амплитуды остальных колеблются в диапазоне от 0,2 до 0,5 .

Далее произведем оценку математического ожидания и дисперсии оценки пеленга, получаемые в пределах периода $T K$, для линейной антенны с апертурой 0,5 км. Так как разность фазовых скоростей и количество нормальных волн зависит от частоты, период $T K$ вычисляется по следующему выражению

$$
T K=\max \left(\frac{2}{f \cdot\left(\frac{1}{c_{i}}-\frac{1}{c_{j}}\right)}\right),
$$

где $i, j$ номера нормальных волн, принимающих значения $1,2, \ldots, N m$ и $i \neq j, N m$ - количество нормальных волн на частоте $f$.

Строго говоря, период интерференции при наличии нескольких нормальных волн не соответствует выражению (11), однако, как отмечалось в [14], таким образом можно вычислить квазипериод интерференции, а изменения от квазипериода к квазипериоду можно считать незначительными.
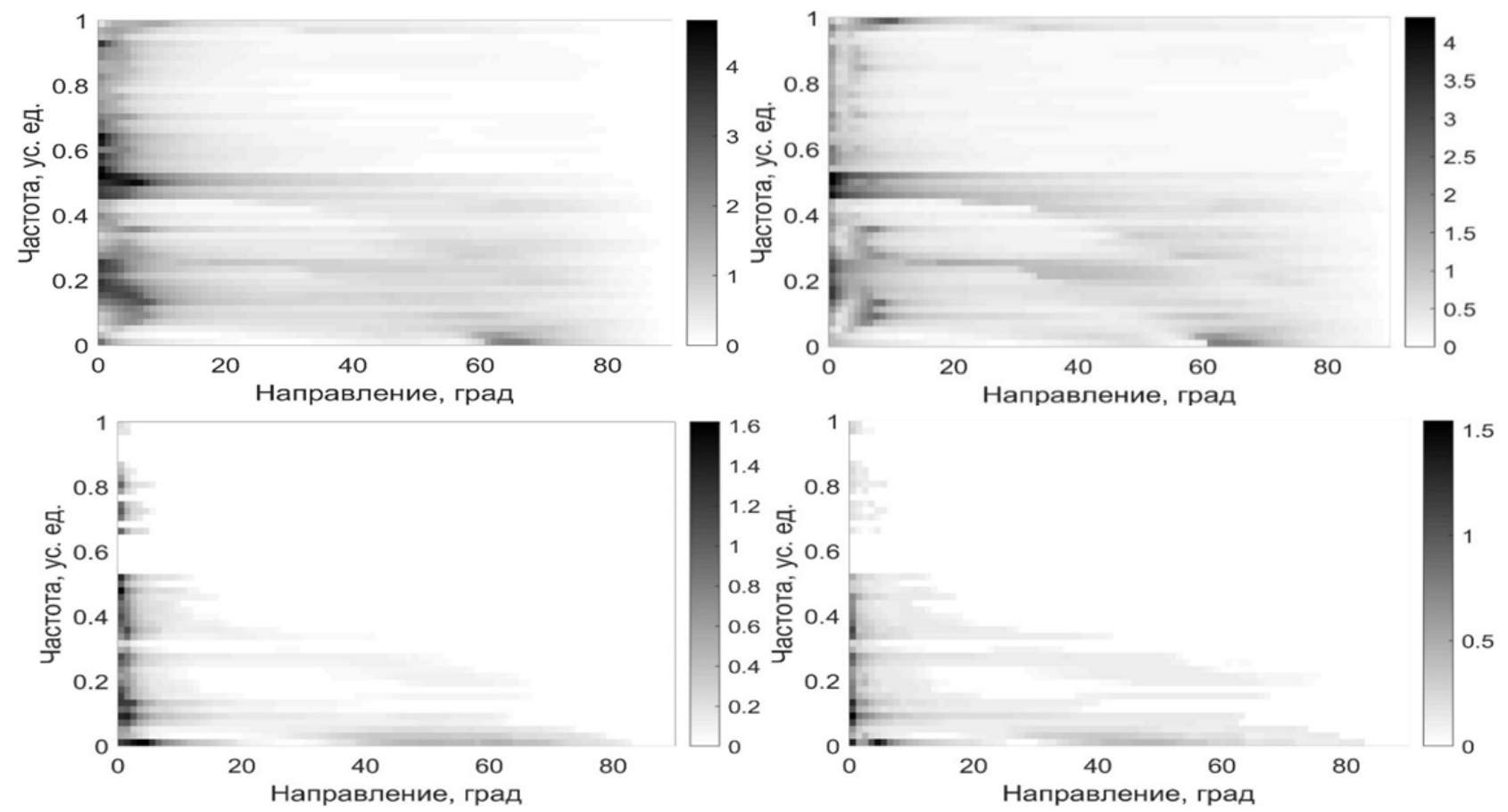

Рис. 5. Математическое ожидание (колонка слева) и СКО (колонка справа) ошибок пеленга для 0,5 км антенны, в зависимости от частоты и направления прихода волны, полученные с использованием алгоритмов А-Г (сверху вниз). Шкала цветности представлена в градусах.

Для лучшей визуализации максимальная ошибка пеленга на рисунках была ограничена по уровню $10^{\circ}$. Верхнюю гранииу следует воспринимать как $\geq 10^{\circ}$ 

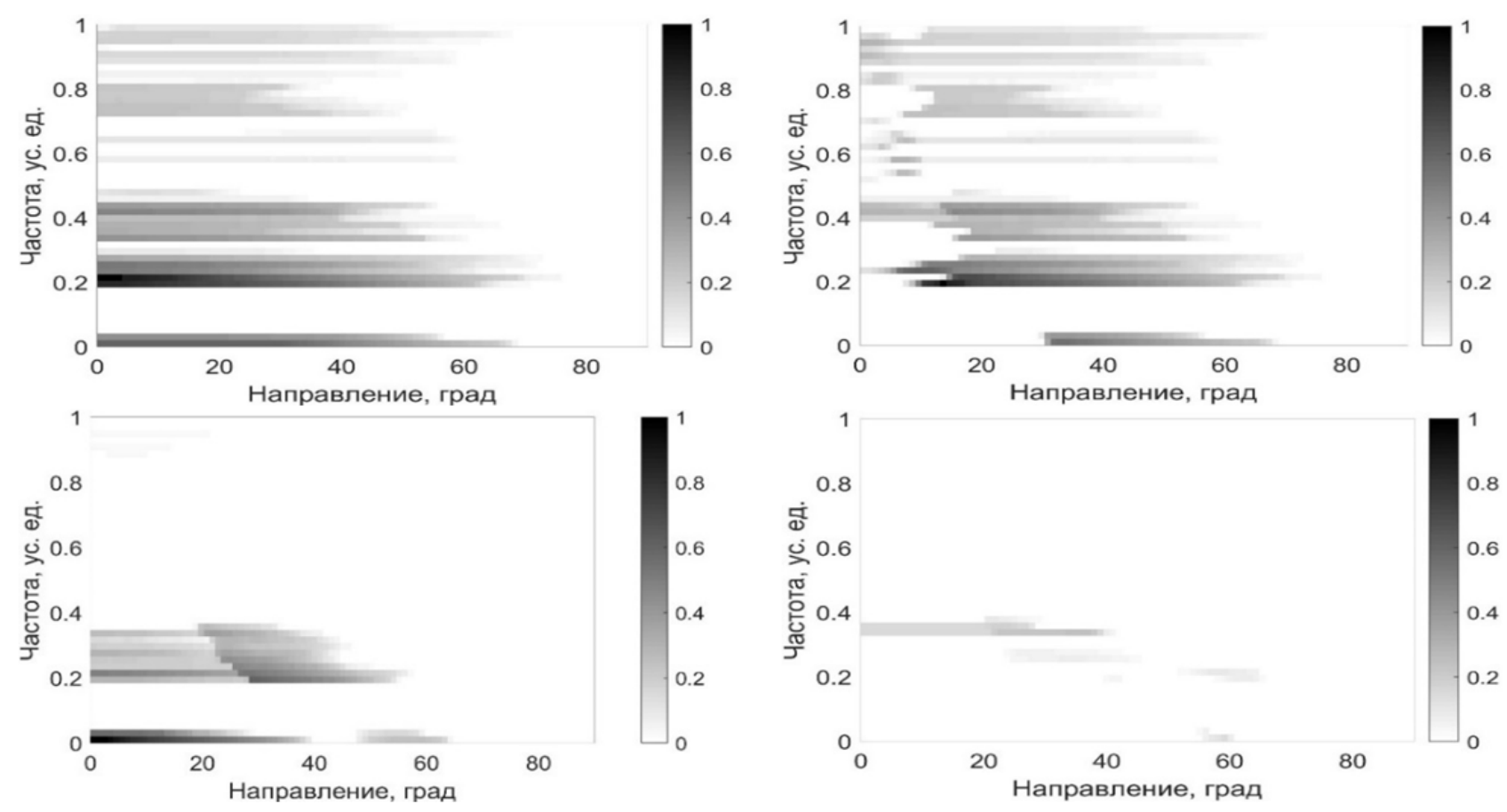

Рис. 6. Вероятность появления ложной отметки для алгоритмов А и Б (верхние) и В и Г (нижние)

2. Чем ниже рабочая полоса частот, тем меньше разрешение антенны и тем больше ошибки пеленгации.

3. Чем ближе направление прихода сигнала к направлению бегущей волны, тем больше ошибки. В направлениях близких к нормали антенны все алгоритмы одинаково эффективны.

4. Несмотря на реализацию полностью согласованной обработки (Г), из-за недостаточного разрешения нормальных волн, при вычислении $|D(\theta)|^{2}$ ошибка определения пеленга не нулевая, но стремится к нулю. Однако, заметим, что при вычислении коэффициента корреляции между компенсатором и волновым пакетом алгоритм Г безошибочно определяет пеленг.

При работе с реальными антеннами количество источников сигнала априорно неизвестно, поэтому при наличии нескольких значимых максимумов в отклике антенны, будут появляться ложные отметки. Рассмотрим какова вероятность того, что отдельные нормальные волны будут разрешаться и давать в отклике антенны несколько значимых максимумов.

Вероятность появления ложной отметки рассчитывалась как отношение количества случаев с несколькими разрешенными значимыми максимумами к общему количеству случаев $n$. Рассматривая рис. 6 можно отметить, что алгоритмы А и Б довольно часто при любом рабочем диапазоне частот будут давать как минимум одну ложную отметку первичного обнаружения морского объекта. По мере усложнения алгоритма вероятность появления ложной отметки уменьшается, так как энергия разных нормальных волн собирается в одном направлении и прочие максимумы в отклике антенны становятся незначимыми.

\section{ЗАКЛЮЧЕНИЕ}

В условиях мелкого моря, в низкочастотном диапазоне дисперсия фазовых скоростей нормальных волн большая и отклики линейной антенны на нормальные волны могут занимать сектор направлений шириной до $35^{\circ}$, что приводит к неоднозначности оценки пеленга, снижению эффективности работы антенны и созданию слепых зон. В данной работе показано, что несмотря на точные априорные знания о среде распространения сигналов, применение известных оптимальных и квазиоптимальных алгоритмов шумопеленгации, при большом отношении с/ш и отсут- 
Моделирование и анализ точности алгоритмов шумопеленгации источников низкочастотных ...

ствии других мешающих факторов, связанных с характеристикой источника звука, не позволяют получить точный пеленг.

На точность пеленгования сильно влияет способность антенны разрешать нормальные волны. Приведённый анализ показал, что значение ошибок пеленга зависит от степени перекрытия основных лепестков откликов антенны на отдельные нормальные волны, разности фазовых скоростей нормальных волн, их амплитуд и начальных фаз, а также от длины апертуры антенны.

На основании проведённого анализа и результатов моделирования сделаны выводы о том, что: по мере увеличения сложности алгоритма (от А к Г) математическое ожидание и СКО ошибок пеленга уменьшаются; чем ниже рабочая полоса частот, тем меньше разрешение антенны и тем больше ошибки пеленгации; чем ближе направление прихода сигнала к направлению бегущей волны, тем больше ошибки пеленгации. В направлениях близких к нормали антенны все алгоритмы одинаково эффективны с точки зрения определения пеленга.

Отмечается, что каждая разрешенная нормальная волна представляет собой максимум в отклике антенны, в связи с чем при обнаружении источника звука каждый максимум может быть воспринят как отдельный источник. В связи с этим появляются ложные отметки первичного обнаружения.

В случаях, когда разрешение значимых по амплитуде нормальных волн отсутствует, целесообразно применять алгоритм, настроенный на одну «среднюю» нормальную волну.

Соотношения, приведенные в работе, можно использовать при синтезе методов и алгоритмов оценки фазовых скоростей нормальных волн по непрерывному гидроакустическому сигналу, в условиях не полного разрешения.

Сделанные выводы позволяют говорить о том, что ГАС необходимо разрабатывать с учётом района, в котором их собираются использовать. В противном случае рассмотренные алгоритмы шумопеленгации и ГАС теряют часть своей эффективности. Однако, для разрешения нормальных волн может по- требоваться использование антенны с длиной апертуры в несколько километров, что реализовать на практике не всегда возможно. В этом случае необходимо говорить о необходимости разработки или модификации квазиоптимальных алгоритмов шумопеленгации, которые учитывали бы взаимодействие нормальных волн, уменьшали СКО ошибок пеленгации и делали оценку пеленга несмещенной.

\section{СПИСОК ЛИТЕРАТУРЫ}

1. Елисеевнин, В. А. Использование интерференционной структуры звукового поля в волноводе для определения направления на источник / В. А. Елисеевнин // Акуст. журн. 2001. - T. 47, № 3. - С. 341-345.

2. Елисеевнин, В. А. Усредненный отклик горизонтальной линейной антенны в мелком море / В. А. Елисеевнин // Акуст. журн. 2004. - Т. 50, № 2. - С. 193-197.

3. Кузнецов, Г. Н. О возможности применения модели с эквивалентной плоской волной для повышения точности пеленгования низкочастотных сигналов в мелком море / Г. Н. Кузнецов, О. В. Лебедев // Акуст. журн. 2012. - T. 58, № 5. - С. 628-638.

4. Low frequency beamforming in shallow water environments / Shima Abadi [et al] // JASA. 2016. - V. 30, № 1. - P. 1-10.

5. Мальщшкин, Г. С. Оптимальные и адаптивные методы обработки гидроакустических сигналов. Т. 1. Адаптивные методы / Г. С. Малышкин. - СПб. : ОАО «Концерн «ЦНИИ «Электроприбор», 2011. - 374 с.

6. Сазонтов А. Г. Согласованная пространственная обработка сигналов в подводных звуковых каналах (обзор) / А. Г. Сазонтов, А. И. Малеханов // Акуст. журн. - 2015. T. 61, № 2. - С. 233-253.

7. Машошин, А. И. Практические задачи гидроакустики, решаемые с использованием алгоритмов обработки сигналов, согласованных со средой их распространения (обзор) / А. И. Машошин // Фундаментальная и прикладная гидрофизика. - 2017. - Т. 10, № 1. C. 37-48. 


\title{
Ю. В. Махнев
}

8. Мальшкин, $\quad$ Г. С. Оптимальные и адаптивные методы обработки гидроакустических сигналов. Т. 1. Оптимальные методы / Г. С. Малышкин. - СПб. : ОАО «Концерн «ЦНИИ «Электроприбор», 2009. - 400 с.

9. Смарышев, М. Д. Гидроакустические антенны / М. Д. Смарышев, Ю. Ю. Добровольский. - Л. : Судостроение, 1984. - 304 с.

10. Ревинская, О. Г. Сложение колебаний. Биения / О. Г. Ревинская, Н. С. Кравченко // Томский политехнический университет. Томск : Изд-во Томского политехнического университета, 2015. - 21 с.

11. Сергиенко, А. Б. Цифровая обработка сигналов / А. Б. Сергиенко. - СПб. : Питер, 2003. $-604 \mathrm{c}$.

12. Махнев, Ю. В. О возможности реализации согласованной со средой обработки для низкочастотного гидроакустического волновода / Ю. В. Махнев, А. Т. Трофимов // Прикладные технологии гидроакустики и гидрофизики: сб. тр. XIV Всеросс. конф. Санкт-Петербург : 2018. - С. 480-482.

13. Гринюк, А. В. Анализ пространственно-временных характеристик низкочастотных гидроакустических сигналов в мелком море / А. В. Гринюк, В. Н. Кравченко, Ю. В. Махнев, А. Т. Трофимов // Прикладные технологии гидроакустики и гидрофизики: сб. тр. XIII Всеросс. конф. - Санкт-Петербург : 2016. - С. 376-378.

14. Исакович, М. А. Общая акустика. Учебное пособие. / М. А. Исакович. - Издательство «Наука», Главная редакция физико-математической литературы, Москва, 1973. - 495 с.

Махнев Юрий Владимирович - аспирант государственного университета «Дубна», научный сотрудник АО «НИИ «Атолл», Дубна, Россия. E-mail: mahnevyu@yandex.ru

\section{MODELING AND ANALYSIS OF ACCURACY OF ALGORITHMS OF LOW FREQUENCY BEAMFORMING IN SHALLOW SEA}

\author{
Y. V. Makhnev \\ JSC «Scientific and Research Institute «Atoll»
}

\begin{abstract}
Annotation. The use of spatial processing of low-frequency hydroacoustic signals in the shallow sea gives direction finding errors associated with the multimode nature of the propagation of hydroacoustic signals. To reduce direction finding errors, it is necessary to use noise direction finding algorithms that are matched with waveguide. Due to the complexity of their implementation, adaptive or quasi-optimal algorithms are used in practice. However, with a large dispersion of the phase velocities of normal waves and a receiving antenna with low resolution, the efficiency of the algorithms decreases. The article contains modeling of several well-known noise direction finding algorithms, including matched-field processing. The dependence of the accuracy of bearing in the application of different algorithms on the ability of the hydroacoustic system to resolve normal waves was investigated. It is shown that their resolution depends on the interference period, antenna length and initial phases of normal waves. Formulas and relations allowing to estimate the magnitude of the bearing determination error are given. The simulation of the hydroacoustic system with specific parameters is carried out. The parameters of the waveguide were set close to real. The mean value and the mean square deviation of the direction finding errors and the probability of false detection of the sound source were calculated.

Keywords: hydroacoustics, matched-field processing in low-frequency range, robust algorithms, shallow sea, linear antennas, beamforming, noise direction, resolution of signals.
\end{abstract}

Makhnev Yuriy Vladimirovich - Postgraduate Student, Dubna State University, researcher, JSC «Scientific and Research Institute «Atoll», Dubna, Russia. E-mail: mahnevyu@yandex.ru 